\title{
APLICAÇÕES DA TECNOLOGIA DE MEMBRANAS NO PROCESSAMENTO DE ÓLEOS VEGETAIS
}

\author{
ANA PAULA BADAN RIBEIRO* \\ MARINALDA DA SILVA SOARES** \\ JULIANA MARIA LEITE NÓBREGA DE MOURA*** \\ MILUSKA CASTILHO CÁCERES ${ }^{* * * *}$ \\ LIRENY APARECIDA GUARALDO GONÇALVES ${ }^{* * * *}$
}

\begin{abstract}
Esta revisão de literatura teve como objetivo relatar as pesquisas que têm sido realizadas sobre a aplicação da tecnologia de membranas na área de óleos vegetais, com ênfase particular nas etapas de degomagem, desacidificação e remoção de pigmentos. Estudos sobre a concentração de tocoferóis também foram abordados. $O$ processamento de óleos através de membranas, efetivado em baixas temperaturas e com eliminação de diversas etapas frente aos métodos convencionais, apresenta-se como alternativa promissora, refletindo a tendência mundial por processos menos dispendiosos e tecnologicamente mais avançados.
\end{abstract}

PALAVRAS-CHAVE: ÓLEOS VEGETAIS - PROCESSAMENTO; TECNOLOGIA DE MEMBRANAS; ULTRAFILTRAÇÃO.

\section{INTRODUÇÃO}

Os óleos de soja, palma, canola e girassol destacam-se em termos de volume de produção mundial. No ano de 2003, a produção

\footnotetext{
* Doutoranda em Tecnologia de Alimentos, Laboratório de Óleos e Gorduras, Departamento de Tecnologia de Alimentos (DTA), Faculdade de Engenharia de Alimentos (FEA), Universidade Estadual de Campinas (UNICAMP) (e-mail: badanribeiro@yahoo.com.br).

** Pesquisadora, Doutora em Tecnologia de Alimentos, Departamento de Engenharia Química e de Alimentos, Universidade Federal do Pará (UFPA).

*** Doutoranda em Tecnologia de Alimentos, Laboratório de Óleos e Gorduras, DTA, FEA, UNICAMP.

**** Doutoranda em Tecnologia de Alimentos, Laboratório de Óleos e Gorduras, DTA, FEA, UNICAMP.

**** Professora, Doutora em Ciência de Alimentos, Laboratório de Óleos e Gorduras, DTA, FEA, UNICAMP.
} 
mundial de óleo de soja situou-se em torno de 200 milhões de toneladas (USDA, 2004). Aproximadamente $80 \%$ dos óleos vegetais são usados em alimentos e envolvem óleos para saladas, fritura, maionese e margarinas. Os $20 \%$ restantes são dirigidos para aplicações industriais que incluem detergentes, cosméticos, lubrificantes, tintas, vernizes e plásticos (HATJE, 1989).

Óleos vegetais brutos são constituídos por mais de $95 \%$ de triacilgliceróis e por compostos minoritários como fosfolipídios, traços de carboidratos, ácidos graxos livres e produtos de degradação dos ácidos graxos. Além disso, na fração correspondente à matéria insaponificável, estão presentes os esteróis, tocoferóis,carotenóides, hidrocarbonetos e ceras. Logo, torna-se necessário submeter o óleo bruto ao processo de refino para que o mesmo adquira características desejáveis (HUI, 1996).

O refino do óleo bruto, visando remover ou reduzir compostos minoritários indesejáveis, constitui procedimento extensivo com grande número de estágios. Devido à importância econômica do refino, grandes esforços têm sido realizados para melhorá-lo e simplificá-lo.

O interesse na aplicação de membranas em tecnologia de lipídios tem crescido bastante nos últimos anos. Sua utilização melhora o processo de produção, reduz o consumo de energia e a produção de águas residuais com a possibilidade de ausência de insumos químicos e de fácil mudança de escala (SNAPE e NAKAJIMA, 1996).

O processamento de óleos por membranas pode ser considerado como alternativa fortemente promissora, visando processos menos dispendiosos, tecnologicamente superiores e que preservam as características nutricionais da matéria-prima.

O objetivo desta revisão foi avaliar o potencial das aplicações da tecnologia de membranas na indústria de óleos vegetais com destaque para processos específicos da área de lipídios e para os parâmetros de desempenho das membranas, buscando analisar a possibilidade da transferência dessa tecnologia ao setor produtivo.

\section{PROCESSAMENTO CONVENCIONAL DE ÓleOS VEGETAIS COMESTÍVEIS}

A etapa inicial do processamento de óleos vegetais consiste na extração 
de óleos a partir de sementes oleaginosas. O tipo de extração depende do teor inicial de óleo do material a ser processado. Para matérias-primas com teor de óleo inferior a $20 \%$ como, por exemplo, a soja (e a torta extraída pela prensagem mecânica) utiliza-se a extração por solvente. Essa origina uma miscela óleo/solvente, que deve ser submetida posteriormente ao processo de destilação para obtenção do óleo bruto livre de solvente (SNAPE e NAKAJIMA, 1996).

O processo convencional de refino químico consiste nas etapas de degomagem, neutralização, clarificação e desodorização (HOFFMAN, 1989). A degomagem, primeira etapa do processo de refino de óleos vegetais, visa a remoção de fosfolipídios pela adição de agentes hidratantes (água/ácido) para permitir a precipitação e remoção de lecitinas em centrífugas separadoras. Essa etapa, realizada na unidade de esmagamento, tem por finalidade a operacionalização de estocagem e transporte do óleo bruto às unidades fabris de refino, normalmente distantes das unidades de esmagamento. A ausência dessa etapa para óleos com altos teores de fosfolipídios invibializaria o transporte pelo fato de ocasionarem entupimentos em tanques e ductos. Após a degomagem, o óleo é seco e estocado ou transportado a granel (ERICKSON, 1995a).

O óleo degomado, na unidade de refino, é tratado com solução alcalina de hidróxido de sódio para reagir e precipitar os ácidos graxos livres como sabões e, ao mesmo tempo, remover traços remanescentes de fosfolipídios. As unidades de refino otimizam a eliminação de fosfolipídios não-hidratáveis e traços de metais com adição de ácido fosfórico previamente ao tratamento com soda para evitar a formação de emulsões. O uso de soluções cáusticas para remoção de não-glicerídios indesejáveis e ácidos graxos livres é efetivo, mas pode ocasionar a saponificação de lipídios neutros e o arraste dos mesmos pelos sabões formados (KOSEOGLU e ENGELGAU, 1990; ERICKSON, 1995b).

O óleo neutro, lavado e seco pode ainda conter pequenas quantidades de impurezas que precisam ser removidas para obtenção de produto com cor e sabor aceitáveis ao consumo final (GUNSTONE, 1983).

A clarificação de óleos vegetais consiste na adição de terras clarificantes, ativadas ou naturais, ao óleo neutralizado. Resumidamente, as funções do branqueamento envolvem a remoção ou redução dos níveis dos pigmentos (cor), de sabões, dos produtos de oxidação, de traços de metais e dos fosfolipídios. A ação da terra clarificante torna-se mais eficiente em meio anidro. Portanto, a primeira etapa do branqueamento 
é a secagem do óleo, já que o óleo proveniente da etapa de neutralização e lavagem contém sempre umidade (ERICKSON, 1995c).

A etapa final do processamento de óleos vegetais é a desodorização, que visa a remoção de compostos com sabor e odor indesejáveis, a redução do conteúdo de ácidos graxos livres, a destruição de peróxidos que comprometem a estabilidade do óleo e de pigmentos termossensíveis (como os carotenóides). Consiste no processo de destilação por arraste de vapor, realizado em altas temperaturas e alto vácuo (BELITZ e GROSCH, 1997).

\section{PROCESSO ALTERNATIVO: TECNOLOGIA DE MEMBRANAS}

\subsection{INTRODUÇÃO AOS PROCESSOS COM MEMBRANAS}

Os processos de separação com membranas destacam-se como alternativas aos processos convencionais de separação nas indústrias químicas, farmacêuticas, biotecnológicas e de alimentos. Em muitos casos, o baixo consumo de energia, a redução no número de etapas do processamento, maior eficiência na separação e maior qualidade do produto final são seus principais atrativos (STRATHMANN, 1990).

O fator que distingue os processos mais comuns de separação por membranas (microfiltração, ultrafiltração, nanofiltração e osmose reversa) é a aplicação de pressão hidráulica para acelerar o processo de transporte. Entretanto, a natureza da membrana controla quais componentes permearão e quais ficarão retidos, uma vez que os mesmos são diferencialmente separados de acordo com seus pesos molares ou tamanho de partícula (CHERYAN, 1998).

O mecanismo de separação ocorre, na prática, de maneira mais complexa devido à influência de parâmetros como composição da membrana, configuração do equipamento, superfície da membrana, pressão, temperatura e dinâmica do fluido (KOSEOGLU, RHEE e LUSAS, 1989).

No sistema de filtração convencional, o escoamento do fluido (líquido ou gasoso) é perpendicular à superfície da membrana. Isso provoca 0 depósito dos solutos sobre a mesma, sendo necessária a interrupção do processo para limpeza ou substituição do filtro. Na filtração tangencial por membranas, o escoamento do fluido é paralelo à superfície da membrana. Devido à alta velocidade ocorre o arraste dos solutos que tendem a se acumular na superfície, o que torna esse processo mais 
eficiente (PAULSON, WILSON e SPATZ, 1984). A Figura 1 representa a comparação entre os dois sistemas de filtração.

Normalmente, usa-se gradiente de pressão hidrostática como força matriz para atingir o fluxo hidrodinâmico desejado. Em alguns casos, os gradientes de concentração e o potencial elétrico podem ser usados como forças motrizes adicionais (ROSENBERG, 1995).

\section{FIGURA 1 - DIFERENÇA ENTRE A FILTRAÇÃO PERPENDICULAR (CONVENCIONAL) E A FILTRAÇÃO TANGENCIAL}

Filtraçāo Perpendicular
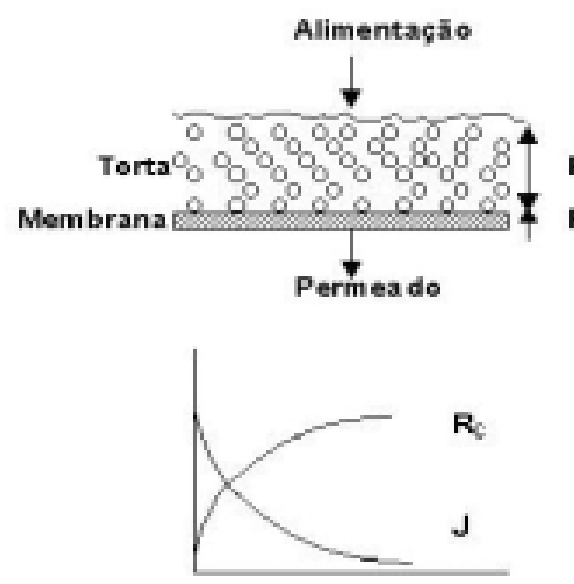

Tempo
Filtraçāo Tangencial
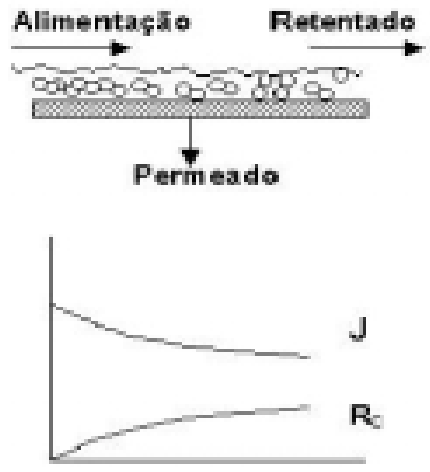

Tempo

$R_{C}$ é a resistência da torta formada na membrana devido aos solutos impermeáveis, $R_{M}$ é a resistência da membrana, J é o fluxo (CHERYAN, 1998).

Segundo SNAPE e NAKAJIMA (1996) são utilizadas na microfiltração pressões inferiores a 0,2 Mpa, separando moléculas entre 0,025 e $10 \mu \mathrm{m}$. A ultrafiltração envolve pressões superiores a $1 \mathrm{MPa}$ e separação de partículas com peso molecular entre 1 e $300 \mathrm{Kda}$. Na nanofiltração são utilizadas pressões entre 1 e $4 \mathrm{MPa}$ para separação de partículas com peso molecular entre 350 e $1000 \mathrm{Da}$. Já na osmose reversa são utilizadas pressões entre 4 e $10 \mathrm{MPa}$ e concentração de partículas com peso molecular menor que $350 \mathrm{Da}$. 


\subsection{MEMBRANAS}

A principal função da membrana é atuar como barreira seletiva, permitindo a passagem de certos componentes de uma mistura e retendo outros. Sua seletividade está relacionada com as dimensões da molécula ou partícula de interesse de separação, ao tamanho do poro, à difusividade do soluto na matriz e às cargas elétricas associadas (CHERYAN, 1998).

Escolhe-se o sistema de membranas a partir do tipo de aplicação, devendo-se considerar a porosidade e o coeficiente de retenção da membrana (HABERT, BORGES e NÓBREGA, 1997). Geralmente o tamanho de poro da membrana está indicado na literatura (principalmente pelos fabricantes) mediante a massa molecular de corte (MWC), que designa a massa molar do menor componente que será retido com eficiência de pelo menos $95 \%$. De modo geral, quando se trata da separação de macromoléculas, a unidade mais utilizada para a massa molar de corte é o Dalton. Para o poro de membranas de microfiltração, normalmente maior que o de membranas de ultrafiltração, prefere-se o diâmetro de poro em micrometro ( $\mu \mathrm{m})$ (MODLER, 2000).

\subsubsection{Características das membranas}

As características mais importantes das membranas envolvem a espessura, o diâmetro dos poros (seletividade), a permeabilidade à água e a porosidade. Outras características são os fluxos de permeado e as resistências térmica, química e mecânica (OSTERGAARD, 1989).

A porosidade não deve ser confundida com o tamanho dos poros, pois trata-se da relação entre a parte sólida e os poros da membrana ou a "quantidade de vazios" em sua estrutura (porosidade global). Pode ser relativa à parte filtrante da membrana (pele filtrante) e, nesse caso, é expressa em poros $/ \mathrm{cm}^{2}$ (densidade dos poros). Quanto maior a porosidade da subcamada, menor será a resistência ao fluxo de solvente através da membrana. A determinação do diâmetro de poros da membrana assume grande importância para sua caracterização (CHERYAN, 1998).

\subsubsection{Tipos de membranas}

As membranas sintéticas comerciais são produzidas a partir de duas classes distintas de material. Os polímeros, constituídos de material orgânico como acetato de celulose, poliamidas, polissulfonas, polifluoreto de vinilideno dentre outros, e os inorgânicos, como metais e materiais 
De acordo com a evolução tecnológica, as membranas podem ser classificadas em três classes distintas. Na primeira geração estão as membranas derivadas de acetato de celulose que foram desenvolvidas originalmente para dessalinização da água do mar. São sensíveis ao pH $(3-8)$ e a temperatura (máximo $50^{\circ} \mathrm{C}$ ) e susceptíveis aos microrganismos e desinfetantes (CHERYAN, 1998). As da segunda geração são elaboradas com polímeros sintéticos, principalmente derivados de polissulfona ou poliolefina. Foram introduzidas a partir de 1975, com diferentes composições químicas e propriedades funcionais, tais como as de poliamidas e polibenzimidazóis que são resistentes à hidrólise e ao ataque de microrganismos, porém degradam na presença de cloro (ZANINI, 1994). As de terceira geração são membranas constituídas de material cerâmico a base de óxido de zircônio ou alumina depositados sobre superfície de grafite ou outros materiais. Apresentam grande resistência mecânica, suportam altas pressões, toleram toda faixa de $\mathrm{pH}\left(0\right.$ a 14) e temperaturas superiores a $400^{\circ} \mathrm{C}$. São quimicamente inertes e de custo muito elevado (SUTHERLAND e FREE, 1991).

\subsubsection{Fenômenos envolvidos no processo}

Durante o processo de separação por membranas, geralmente ocorre declínio do fluxo de permeado (a pressão constante) com o tempo. Os principais problemas relacionados com esse comportamento envolvem a formação da camada de gel, a polarização por concentração e a colmatagem. A formação da camada de gel ocasiona prejuízo no funcionamneto hidrodinâmico do sistema, pois constitui mais uma barreira para o fluxo de permeação. Essa camada de gel ocorre quando há precipitação de macromoléculas na superfície da membrana. A colmatagem resulta da penetração de solutos presentes em soluções de macromoléculas ou de suspensões coloidais nos poros da membrana, acarretando também diminuição no fluxo do solvente e alterando as características de retenção. Além disso, durante o processo ocorre acúmulo de solutos próximo à superfície da membrana por transporte convectivo, sendo parte do solvente removida do fluido, o que ocasiona maior concentração de solutos na superfície da membrana em relação à da solução. Esse aumento na concentração de solutos na superfície da membrana, conhecido como polarização da concentração, é responsável pela diferença observada entre o fluxo de permeado final e inicial comparado com o fluxo de água pura (CHERYAN, 1998). 


\subsubsection{Parâmetros operacionais}

Os principais parâmetros de operação que afetam o fluxo de permeado são a pressão, a temperatura, a concentração de alimentação e a velocidade tangencial.

O fluxo de permeado é diretamente proporcional à pressão aplicada e inversamente proporcional à viscosidade. A viscosidade pode ser controlada pela concentração de sólidos na alimentação e pela temperatura. Sob condições restritas, a elevação da pressão ou da temperatura acarreta aumento no fluxo de permeado. Entretanto, a utilização de pressões elevadas pode ocasionar a compactação da camada de gel, aumentando a colmatagem da membrana (HWANG e KAMMERMEYER, 1998).

O aumento na concentração de alimentação afeta a viscosidade, a densidade e a difusividade da solução de alimentação, ocasionando decréscimo no fluxo de permeado. Em geral, temperaturas mais elevadas proporcionam maiores fluxos de permeados em regiões em que existe controle de pressão e de transferência de massa. O efeito da temperatura decorre da redução ocasionada na viscosidade do fluido e na mobilidade das moléculas, ou seja, na difusividade. O aumento da velocidade tangencial incrementa a taxa de permeação por provocar maior turbulência. A turbulência, proveniente de agitação ou bombeamento do fluxo, promove a desorganização na concentração de solutos na superfície da membrana e reduz a espessura da camada limite. Esse constitui um dos métodos mais simples e efetivos de se controlar o efeito da polarização da concentração (STRATHMANN, 1990).

A configuração também afeta o desempenho das membranas. Alto desempenho quanto ao fluxo de permeado e retenção do soluto desejado deve ser balanceado pela sua maior propensão à colmatagem, custo, facilidade de limpeza e substituição (PORTER, 1990).

\subsubsection{Parâmetros importantes para a análise do desempenho e eficiência do processo de ultrafiltração}

Para a análise do desempenho e eficiência do processo de ultrafiltração os seguintes parâmetros são fundamentais (RENNER e SALAM, 1991):

- fluxo de permeado $(F)$ = quantidade obtida de permeado em $\mathrm{kg}$ por $\mathrm{m}^{2}$ de membrana por hora $\left(\mathrm{kg} \cdot \mathrm{m}^{-2} \cdot \mathrm{h}^{-1}\right)$; 
- coeficiente de retenção = fornece medida quantitativa da capacidade da membrana em reter, por exemplo, as moléculas de fosfolipídios sob determinadas condições de operação. É dado em porcentagem: $R=(1-\mathrm{Cp} / \mathrm{Ci})^{*} 100$, na qual $\mathrm{Ci}$ e Cp são, respectivamente, a concentração inicial de fosfolipídios no óleo e a concentração de fosfolipídios no permeado em cada amostragem;

- fator de concentração $(\mathrm{Fc})$ = quantifica a redução de massa atingida pela ultrafiltração. É calculado como a razão entre a massa inicial na alimentação e a massa final de retentado: $F c=M_{A} / M_{R}$, na qual $M_{A}=$ massa na alimentação e $M_{R}=$ massa no retentado;

- pressão transmembrana $(\mathrm{PTM})=$ média do gradiente da pressão entre o lado do retentado e o lado do permeado, que normalmente está à pressão atmosférica, na entrada e na saída da membrana $\left(\mathrm{P}=\left(\mathrm{P}_{\text {ent }}+\mathrm{P}_{\text {saída }}\right) / 2\right)$.

\section{APLICAÇÃO DE MEMBRANAS EM TECNOLOGIA DE ÓLEOS VEGETAIS}

As pesquisas sobre membranas no processamento de óleos vegetais iniciaram-se no final da década de 80 , e ainda se encontram em fase de estudos laboratoriais.

O processo convencional de refino de óleos caracteriza-se por altos requerimentos energéticos, perdas de óleo neutro, necessidade de grandes quantidades de água e insumos químicos, perda de nutrientes e elevada produção de efluentes. Em contraste, o processamento mediante membranas mostra-se notadamente mais simples. Oferece vantagens em relação ao processo convencional devido ao baixo consumo energético, operação em temperaturas amenas, ausência de adição de produtos químicos e retenção de nutrientes e outros compostos desejáveis (SUBRAMANIAN et al., 2001a).

Estudos sobre a viabilidade econômica do processo aplicado à industria de óleos não existem. Dados de 1990 relatam que a substituição ou complementação do refino convencional pela tecnologia de membranas poderia acarretar economia de 15 a 21 trilhões Btu/ano, sendo 2 trilhões $\mathrm{Btu} / \mathrm{ano}$ correspondentes à recuperação do solvente. Adicionalmente, 0 decréscimo nas perdas de óleo neutro e nos requerimentos de terra clarificante apresenta-se como vantagem potencial dessa alternativa tecnológica (KOSEOGLU e ENGELGAU, 1990). 
O processo convencional de refino de óleos vegetais, bem como o refino baseado na tecnologia de membranas, são mostrados na Figura 2.

\section{FIGURA 2 - COMPARAÇÃO ENTRE OS PROCESSOS CONVENCIONAL E DE TECNOLOGIA DE MEMBRANAS}
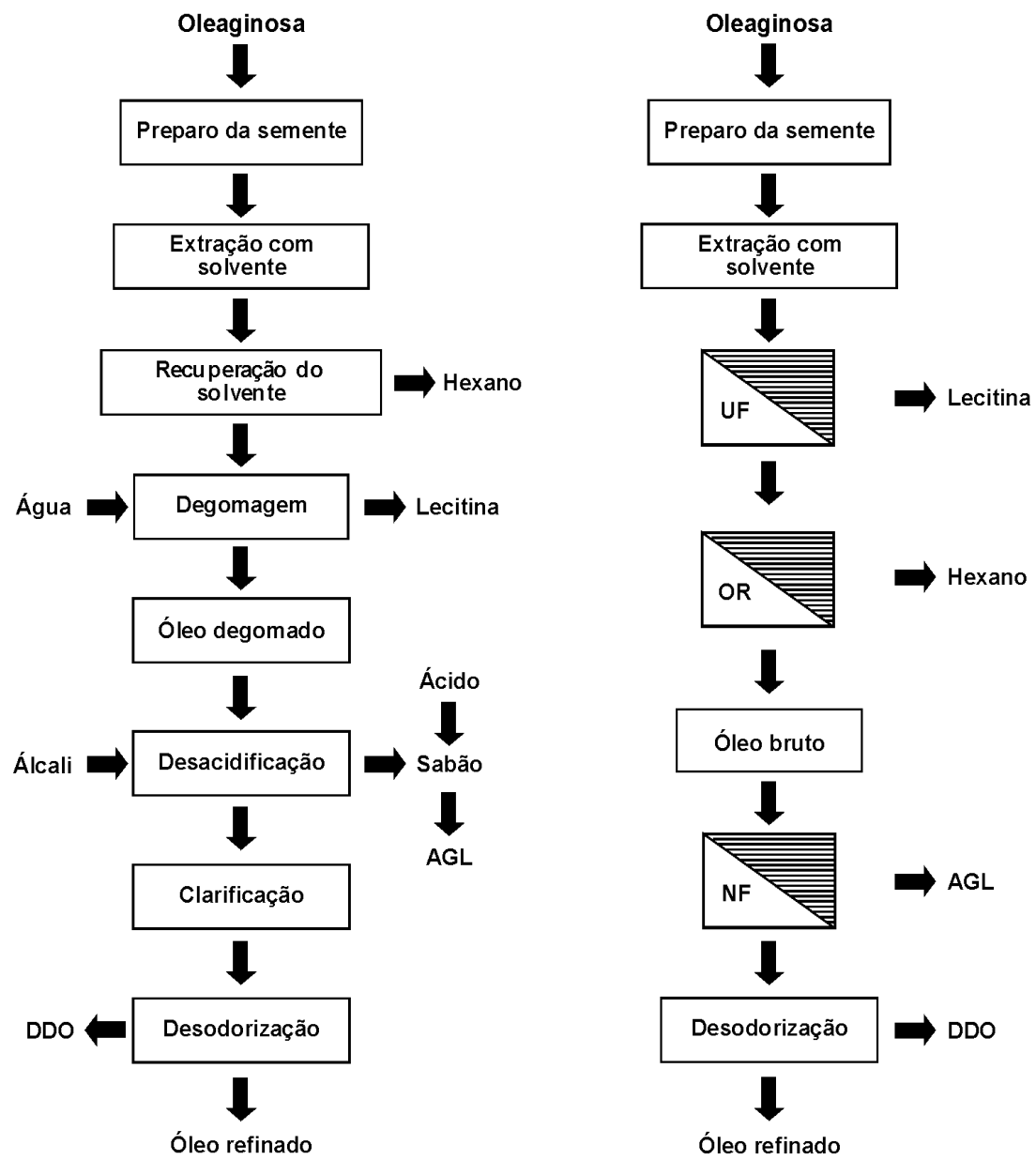

Fonte: CHERYAN (1998).

UF = Ultrafiltração; OR = Osmose Reversa; NF = Nanofiltração; AGL = Ácidos Graxos Livres; DDO = Destilado da Desodorização de Óleo. 


\subsection{DEGOMAGEM}

Para SUBRAMANIAN e NAKAJIMA (1997) é difícil separar triacilgliceróis (massa molar média de $900 \mathrm{Da}$ ) e fosfolipídio (massa molar $800 \mathrm{Da}$ ) por membranas de ultrafiltração devido à similaridade de suas massas molares. Entretanto, os fosfolipídios tendem a formar micelas em meio não-polar de hexano ou óleo.

Segundo PARDUN (1988) micela é a associação de moléculas anfóteras que se agregam. A formação da micela ocorre acima de determinada concentração de monômeros denominada concentração crítica da micela (CMC). O seu tamanho é definido de acordo com sua massa molar e entende-se ainda que está relacionada com a massa molar dos fosfolipídios e com o número de monômeros utilizados para formá-la. A polaridade do solvente utilizado exerce influência significativa sobre a micela de fosfolipídio. Na presença de benzeno, por exemplo, com constante dielétrica igual a 2 Debye, a fosfatidilcolina forma micela com 80 monômeros. O hexano apresenta constante dielétrica de 1,88 Debye (RYDBERG, MUSIKAS e CHOPIN, 1992), próximo do benzeno, caracterizando formação de micela com número de monômeros em alto nível de agregação.

A utilização de membranas no processo de degomagem de óleos vegetais tem sido exaustivamente pesquisada. Diferentes alternativas têm sido propostas, algumas baseadas na remoção de fosfolipídios a partir da miscela (óleo/hexano) e outras na remoção a partir do óleo sem adição de solventes. No primeiro caso, a maior dificuldade envolve a baixa estabilidade das membranas em solventes orgânicos e no segundo caso, o baixo fluxo de permeado como conseqüência da alta viscosidade do óleo (OCHOA et al.,2001).

A aplicação de membranas poliméricas para uso orgânico requer estabilidade química e física frente ao hexano e temperaturas em torno de $50-60^{\circ} \mathrm{C}$. Materiais como poliamida aromática, imida aromática, polifluoreto de vinilideno (PVDF) e politetrafluoretileno parecem ser adequados (IWAMA, 1987). Segundo KOSEOGLU (1991), o processo de degomagem por membranas requer elevadas resistências mecânica, química e térmica. As membranas cerâmicas atendem a esses requisitos e foram introduzidas para aplicação em processos de ultrafiltração, embora a disponibilidade de diferentes tamanhos de poro ainda seja restrita.

Alguns estudos com óleos brutos de origem vegetal foram desenvolvidos 
visando a remoção de fosfolipídios através de membranas em escala laboratorial. DE et al. (1998) e ALICIEO et al. (2002), utilizando membranas cerâmicas em condições diversas de temperatura e pressão (30 a $70^{\circ} \mathrm{C}$; 3 a 50 bar) obtiveram retenções ao redor de $85 \%$, porém com baixos fluxos de permeado $\left(4 \mathrm{~kg} / \mathrm{m}^{2} \mathrm{~h}\right)$. Alternativamente, o uso de membranas poliméricas foi efetuado por SUBRAMANIAN et al. (1998), HASEGAWA et al. (2001) e KORIS e VATAI (2002). Utilizaram pressões variando de 2 a 30 bar e temperaturas entre 40 e $60^{\circ} \mathrm{C}$, o que resultou em menores retenções $(70-78 \%)$, entretanto com melhoria no fluxo de permeado $\left(30 \mathrm{~L} / \mathrm{m}^{2} \mathrm{~h}\right)$.

SUBRAMANIAN et al. (2001a) conduziram estudos exploratórios acerca dos fenômenos de retenção de fosfolipídios em óleos vegetais nos processos com membranas. Basearam-se na caracterização de micelas reversas formadas a partir desses compostos em sistema modelo, contendo óleo de soja sem a adição de solventes. As micelas apresentaram tamanho variando entre 3,56 e 4,80 nm, fato que levou os autores a afirmarem que membrana de natureza hidrofóbica com diâmetro de poro igual a $4 \mathrm{~nm}$ seria a escolha ideal para a separação de fosfolipídios em óleos brutos. No entanto, os fosfolipídios são surfactantes naturais e formam micelas em meio não-aquoso. As micelas formadas apresentaram peso molecular em torno de $20 \mathrm{KDa}$ ou mais e podem ser separadas dos triacilgliceróis pela utilização de membranas adequadas (GUPTA, 1997). Neste sentido, torna-se viável a utilização de miscela óleo/solvente no processo de degomagem por membranas que propicia adicionalmente aumento significativo no fluxo de permeado (10 a $200 \mathrm{~L} / \mathrm{m}^{2} \mathrm{~h}$ ). Fato constatado por diversos estudos (LIN, RHEE e KOSEOGLU, 1997; OCHOA et al., 2001; PAGLIERO et al., 2001; KIM et al., 2002; MOURA et al., 2003; PAGLIERO et al., 2003; PAGLIERO et al., 2004) também em escala laboratorial com membranas poliméricas (massa molar média de corte entre 0,5 a $70 \mathrm{kDa}$ ) resistentes ao hexano. As condições estudadas por esses autores corresponderam a pressões entre 0 a 20 bar, temperaturas de 20 a $50^{\circ} \mathrm{C}$ e teores de óleo em hexano de 20 a $40 \%(\mathrm{~m} / \mathrm{m})$.

Em trabalhos recentes BEl et al. (2004) e SOARES et al. (2004) estudaram, em escala piloto, a degomagem de óleo bruto de soja em miscela $(25 \% \mathrm{v} / \mathrm{v})$, utilizando membranas cerâmicas (alumina) multicanais com diâmetro de poro de $0,01 \mu \mathrm{m}$, a $40^{\circ} \mathrm{C}$, e pressões de 1,2 a 2 bar. Obtiveram retenções ao redor de $99 \%$ e fluxos de permeado variando de 21 a $55 \mathrm{~L} / \mathrm{m}^{2} \mathrm{~h}$. 


\subsection{SEPARAÇÃO DE ÁCIDOS GRAXOS}

KUMAR e BROWMICKD (1996) realizaram, em planta piloto, a nanofiltração de mistura de ácidos graxos, óleo e álcool através de membranas de acetato de celulose, polissulfona e poliamida. Para a membrana de poliamida, com peso molecular médio de corte entre $500 \mathrm{e}$ $600 \mathrm{Da}$, obtiveram fluxo de $67,36 \mathrm{~L} / \mathrm{m}^{2} \mathrm{~h}$ à pressão de 7 bar e os seguintes resultados: $86,82 \%$ de ácidos graxos livres no permeado e $61,71 \%$ de ácidos graxos livres no retentado. Segundo tais autores, a melhor separação dos triacilgliceróis de ácidos graxos livres e álcool ocorreu com poliamidas porque são membranas menos hidrofílicas, resultando em menor compactação.

KALE, KATIKANENI e CHERYAN (1999) reportaram, em escala laboratorial, a desacidificação de óleo bruto de arroz em solvente através de nanofiltração. A concentração de ácidos graxos livres foi reduzida de $16,5 \%$ para 3,7 \% pela extração com metanol na proporção de 1,8:1 metanol/óleo (peso) e foi recuperada por nanofiltração com membranas comerciais (Osmonics, Film Tec). Segundo esses autores, o sistema com dois estágios de membrana pode recuperar $97,8 \%$ dos ácidos graxos livres e resultar em fonte de retentado final com $20 \%$ de ácidos graxos livres, os quais podem ser reciclados em outra extração de ácidos graxos.

ZWIJNENBERG et al. (1999) utilizaram membrana de poliamida (nanofiltração), preparada em laboratório, na separação de dois tipos de mistura (óleo de girassol/acetona e ácidos graxos provenientes do óleo de breu/acetona). Os ensaios foram realizados a 20 bar e $20^{\circ} \mathrm{C}$, com área efetiva de membrana igual a $37 \mathrm{~cm}^{2}$. Misturas contendo óleo de girassol/acetona apresentaram concentrações entre 10 a 50\% (em óleo), resultando na rejeição de triacilgliceróis praticamente constante entre 92-94\%. Estudaram também a rejeição de ácidos graxos livres (AGL) com frações separadas contendo 10\% AGL e 10\% AGL metilados, ambas em acetona. Obtiveram para ambas $48-54 \%$ de rejeição. Segundo tais autores, as moléculas de AGL e AGL metilados diferem significantemente em polaridade, mas não em tamanho. Assim a diferença de tamanho entre triacilgliceróis e AGL consistiria no principal mecanismo de separação entre os mesmos em detrimento da diferença de polaridade.

KOIKE et al. (2002) avaliaram a capacidade de separação de ácidos graxos livres de diferentes tipos de membranas poliméricas comerciais, sem identificação de tamanho de poro (série NTGS - Nitto Denko, Japão). Utilizaram óleo de girassol alto oléico anteriormente submetido à hidrólise 
enzimática, diluído em hexano ou etanol. Os ensaios foram realizados em célula com capacidade de $100 \mathrm{~mL}$ a $30^{\circ} \mathrm{C} \mathrm{e} 40$ bar. O melhor resultado foi obtido com membrana composta silicone/poliamida para misturas óleo/ hexano em que a rejeição de ácidos graxos livres foi de 70,2\%.

\subsection{REMOÇÃO DE PIGMENTOS}

O alto custo de terra de branqueamento, associado com perdas de óleo e subseqüentes problemas de descarte da terra, aumentaram o interesse pela aplicação da tecnologia de membranas para substituir o processo de clarificação tradicional (SNAPE e NAKAJIMA, 1996). Todos os estudos realizados nessa área estão disponibilizados apenas em escala laboratorial.

Experimentos sobre a performance de cor em célula de ultrafiltração de $400 \mathrm{~mL}$ com misturas de óleo - hexano (25\% óleo) foram realizados por KOSEOGLU, RHEE e LUSAS (1989). As leituras de cor do permeado para clorofila e beta- caroteno foram reduzidas em média para $10 \%$ para os óleos brutos de girassol, soja, canola e amendoim. Somente cinco das quinze membranas mostraram-se estáveis ao hexano, mas sua identidade não foi revelada por razões comerciais.

REDDY e NAKAJIMA (1996) verificaram em testes de célula de membranas, a remoção de clorofila e beta-caroteno de óleo de girassol e soja. Membranas de microfiltração de polietileno $(0,03 \mu \mathrm{m})$ apresentaram baixo nível de redução de clorofila $(<4 \%)$ em contraposição à membranas de poliamida que evidenciaram $95 \%$ de redução. Entretanto, para as membranas de poliamida foram verificados baixos fluxos de permeado $\left(0,1\right.$ a $\left.0,2 \mathrm{~kg} \cdot \mathrm{m}^{-2} \mathrm{~h}^{-1}\right)$ com os óleos utilizados. A adição de $50 \%$ de hexano aos mesmos resultou em aumento considerável nos fluxos (200 vezes), embora tenha propiciado menor redução de clorofila (70\%).

Experimentos utilizando óleo de soja bruto, sem a adição de solventes orgânicos, foram realizados por SUBRAMANIAN, NAKAJIMA e KAWAKATSU (1998). Testaram membranas poliméricas compósitas com silicone como camada ativa e polissulfona e poliamida como camadas de suporte, respectivamente. As condições de operação foram mantidas em $40^{\circ} \mathrm{C}$, pressão de 30 bar e agitação de $800 \mathrm{rpm}$. A redução de clorofila no permeado variou de 74 a $80 \%$ para as duas membranas estudadas.

REDDY et al. (2001) estudaram a clarificação de óleo de soja bruto e em solução com hexano ( $50 \%$ p/p) por meio de duas membranas poliméricas 
compósitas (NTGS - 2100 e NTGS - 1100, Nitto Denko, Japão) e uma membrana de polietileno para microfiltração. Os testes foram realizados em célula pressurizada por nitrogênio com agitação de $500 \mathrm{rpm}$ e temperatura de $40^{\circ} \mathrm{C}$. A membrana NTGS -2100 propiciou os melhores resultados com remoção de $96 \%$ e $72 \%$ de clorofila do óleo bruto e da solução óleo/hexano, respectivamente.

SUBRAMANIAN et al. (2001b) avaliaram o mecanismo de rejeição de carotenóides em amostras de óleo de soja bruto e óleo de girassol alto oléico refinado. Utilizaram membrana de poliimida sem identificação de tamanho de poro (NTGS-2200, Nitto Denko, Japão) com $32 \mathrm{~cm}^{2}$ de área efetiva, a 40 bar, 30 a $40^{\circ} \mathrm{C}$ e agitação de $400 \mathrm{rpm}$. Observaram baixa rejeição de carotenos (11 a 20\%), enquanto que as xantofilas (principais carotenóides do óleo de soja) foram rejeitadas em $60 \%$. Também verificaram a ausência de afinidade entre beta-caroteno e os fosfolipídios presentes no óleo.

MOURA et al. (2003) utilizaram membrana de polietersulfona (PES), preparada em laboratório pelo processo de inversão de fase e com ponto de corte de aproximadamente $70 \mathrm{KDa}$, na avaliação de clarificação de óleo de soja bruto. Realizaram a ultrafiltração do óleo bruto e da miscela (óleo/hexano) em célula de filtração perpendicular com agitação e pressurização com $\mathrm{N}_{2}$, a $50{ }^{\circ} \mathrm{C}$. O óleo processado por membranas apresentou redução de $25 \%$ em unidades de amarelo de escala Lovibond. No entanto, tal redução parece estar associada à capacidade adsortiva da membrana, já que com sucessiva ultrafiltração da miscela não houve aumento na retenção. Os autores observaram que como os materiais utilizados para elaboração de membranas podem ser susceptíveis à adsorção de pigmentos, a avaliação da clarificação deve ser efetivada após exaustivo uso das membranas para que os resultados sejam validados.

\subsection{ESTUDO DE TOCOFERÓIS}

Tocoferóis são antioxidantes naturais presentes nos óleos vegetais, que apresentam efeito benéfico sobre a qualidade dos mesmos. Portanto, estudos recentes têm enfocado a aplicação da tecnologia de membranas na preservação ou concentração desses compostos (SUBRAMANIAN et al.,2003).

A permeação preferencial de tocoferóis em relação a triacilgliceróis nos processos com membranas foi primeiramente observada por 
SUBRAMANIAN et al. (1998). Os permeados obtidos apresentaram altos teores de tocoferóis na filtração de óleos de amendoim e de girassol brutos. Utilizaram membrana polimérica preparada em laboratório com silicone como camada ativa e polissulfona e poliimida como camada de suporte, sem descrição das características de poro. Os experimentos foram conduzidos a $30 \mathrm{bar}, 40^{\circ} \mathrm{C}$ e $400 \mathrm{rpm}$. Os óleos de amendoim e girassol apresentaram retenções de tocoferóis de $-60 \%$ e $-96 \%$, respectivamente. De acordo com esses autores, tocoferóis parecem não apresentar afinidade com as micelas de fosfolipídios. $O$ baixo peso molecular desses compostos, aliado a possíveis interações com 0 material da membrana, resultariam em maior permeação (ou em valores negativos de retenção).

Estudos de SUBRAMANIAN, NAKAJIMA e KAWAKATSU (1998) revelaram retenção negativa de tocoferóis (entre-18 e-24\%) para óleo de soja bruto. A filtração em membranas comerciais (contendo silicone como camada ativa e polissulfona e poliimida como camadas de suporte, sem descrição de tamanho de poro) a $30 \mathrm{bar}, 40^{\circ} \mathrm{C}$ e $800 \mathrm{rpm}$ resultou em aumento de $12-15 \%$ desses compostos no permeado. Experimentos com óleo de canola realizados nas mesmas condições elevaram o teor de tocoferóis no permeado em $25 \%$.

SUBRAMANIAN et al. (2003) reportaram a aplicação de membranas densas (não-porosas) no estudo da permeação diferenciada de tocoferóis. Utilizaram membrana com camada ativa de silicone e poliimida como camada de suporte (NTGS-2200, Nitto Denko, Japão). Os experimentos foram realizados com óleo de girassol alto oléico enriquecido com alfatocoferol, com pressão e temperatura variando entre 20 a 50 bar e 20 a $50^{\circ} \mathrm{C}$, respectivamente. Os tocoferóis apresentaram permeação preferencial quando comparados aos triacilgliceróis, correspondendo a valores de rejeição negativos (-30 a $-52 \%)$. Todavia, o aumento na concentração de tocoferóis na alimentação acarretou diminuição da taxa de permeação dos mesmos, embora sem efeito significativo sobre o fluxo total. Os autores não mencionaram as características de poro da membrana.

NAGESHA, SUBRAMANIAN e SANKAR (2003) avaliaram a seletividade de membranas densas com silicone e poliimida como camada ativa e de suporte, respectivamente, quanto à permeação de tocoferóis. Utilizaram amostras de destilado do desodorizado de óleo de soja (DDOS), diluídas ou não em hexano e de destilado do desodorizado de óleo de soja esterificado, sem diluição. Para comparação foram selecionados 
sistemas modelo constituídos por ácido oléico e tocoferóis em diferentes proporções (80:20 e 50:50), diluídos ou não em hexano. Ao sistema modelo isento de hexano adicionaram mistura de ésteres metílicos de ácidos graxos (FAME) de óleo de soja e tocoferóis (90:10). Os experimentos foram conduzidos em célula de filtração pressurizada com nitrogênio a $30 \mathrm{bar}, 30^{\circ} \mathrm{C}$ e $800 \mathrm{rpm}$. O estudo revelou permeação preferencial de tocoferóis em relação aos outros constituintes de baixo peso molecular (ácidos graxos livres e FAME). A seletividade da membrana quanto aos tocoferóis aumentou com o DDOS esterificado. A presença de hexano influenciou positivamente a permeação de tocoferóis por propiciar maior solubilidade desses compostos em relação aos outros constituintes da alimentação. Tais autores afirmaram que os processos com membranas apresentam boas perspectivas futuras no enriquecimento de tocoferóis do DDOS. Entretanto, experimentos em escala piloto seriam necessários para o desenvolvimento do processo.

\section{CONCLUSÃO}

Diante das alterações de ordem econômica e nutricional ocorridas na área alimentícia em geral, a tendência da engenharia de processo na área de óleos e gorduras mostra caminhos alternativos para preservação de compostos importantes existentes em óleos brutos, como os tocoferóis, que apresentam reconhecido valor para a saúde humana. $\mathrm{Na}$ expectativa de preservar grande parte desses nutrientes, o processo através de membranas efetivado em baixas temperaturas e com eliminação substancial de etapas frente aos processos convencionais apresenta-se altamente viável, principalmente, devido total adequação dessa nova tecnologia às questões ambientais.

A aplicação da tecnologia de membranas na área de óleos tem apresentado desenvolvimento crescente, a despeito de dificuldades quanto à disponibilidade de membranas para uso orgânico e estabilidade química das mesmas frente a solventes como o hexano. A membrana ideal para utilização no processamento de óleos vegetais deve combinar requerimentos específicos de retenção com fluxos de permeados adequados aos processos industriais. Na escolha da membrana deve-se portanto, considerar aspectos relacionados com as interações físicoquímicas entre possíveis solventes, materiais constituintes da membrana e componentes presentes no óleo em questão.

Os bons resultados obtidos a partir da degomagem de miscela em escala 
piloto, tanto na redução efetiva do teor de fósforo quanto em fluxos de permeado satisfatórios, evidenciam transferência promissora dessa tecnologia ao setor industrial.

Os processos de desacidificação e clarificação apresentam elevado potencial de exploração, uma vez que os estudos realizados ainda revelam resultados díspares.

\section{Abstract}

\section{APPLICATIONS OF MEMBRANE TECHNOLOGY IN VEGETABLE OIL PROCESSING}

The purpose of this review was to report the research that has been carried out in the application of membrane technology in the area of vegetable oils, with particular emphasis on degumming stage, deacidification and pigments removal. Studies about tocopherols concentration also were reported. The oils processing with membranes, done under low temperatures and with substantial exclusion of stages instead of using conventional method, presents a promising alternative, reflecting the world tendency for less costly but at the same time technically more advanced processes.

KEY-WORDS: VEGETABLE OILS - PROCESSING; MEMBRANE TECHNOLOGY; ULTRAFILTRATION.

\section{REFERÊNCIAS}

1 ALICIEO, T.V.R. et al. Membrane ultrafiltration of crude soybean oil. Desalination, Amsterdan, v.148, p.99-102, 2002.

2 BEI, N. et al. Ultrafiltration of soybean crude oil micelle by ceramic membrane. In: WORLD SOYBEAN RESEARCH CONFERENCE, 6., Foz do Iguaçu, PR, 2004. Abstracts... Londrina: Ed. Embrapa Soja, 2004.

3 BELITZ, H.D.; GROSCH, W. Grasas y aceites comestibles. In: QUÍMICA de los alimentos. Zaragoza: Ed. Acribia, 1997. Cap.14, p.707-708.

4 CHERYAN, M. (Ed.) Ultrafiltration and microfiltration handbook. Lancaster: Technomic Publ., 1998. 526 p.

CUPERUS, F.P.; NIJHUIS, H.H. Applications of membrane technology to food processing. Trends in Food Science \& Technology, v. 7, p. 277-282, 1993.

DE, B.K. et al. Membrane degumming and dewaxing of rice brain oil and its refining. Fett/Lipid, Leinfelden, v.100, n.9, p.416-421, 1998.

ERICKSON, D.R. Degumming and Lecithin Processing and Utilization. In: PRACTICAL handbook of soybean processing and utilization. St. Louis: American Soybean Association / Champaign: American Oil Chemists' Society, 
1995a. p.174-183.

ERICKSON, D.R. Neutralization. In: PRACTICAL handbook of soybean processing and utilization. St. Louis: American Soybean Association / Champaign: American Oil Chemists' Society, 1995b. p.184-202.

ERICKSON, D.R. Bleaching/ Adsorption Treatment. In: PRACTICAL handbook of soybean processing and utilization. St. Louis: American Soybean Association / Champaign: American Oil Chemists' Society, 1995c. p.203217.

GUNSTONE, F.D. Lipids in food chemistry: biochemistry and technology. S.1: London: Pergamon International Library, 1983.

GUPTA, A.K.S. Process for refining crude glyceride oils by membrane filtration. US Patent, n. 4062882, 1997.

HABERT, A.C.; BORGES, C.P.; NOBREGA, R. Aspectos gerais dos processos com membranas. In: HARBERT, A.C.; BORGES, C.P.; NOBREGA, R. Processos de separação com membranas. Rio de Janeiro: Editora da Universidade Federal do Rio de Janeiro, 1997. cap.1.

13 HASEGAWA. F. et al. Processing of corn oil using microfiltration and ultrafiltration membranes. In: CONGRESSO IBEROAMERICANO DE INGENIERIA DE ALIMENTOS, 3. Y CONGRESSO ESPAÑOL DE INGENIERIA DE ALIMENTOS, 1., 2001. Valencia, España. Libro de Resúmenes... Valencia: CIBIA, 2001.

14 HATJE, G. World importance of oils crops and their products. In: OILS crops of the world: their breeding and utilization. Berkshire: Mc Graw Hill, 1989. p. 6-8.

HOFFMAN, G. The chemistry and technology of edible oils and fats and their high fat products. San Diego CA: Academic Press, 1989.

HUI, Y.H. Edible oil and fat products: general applications. In: BAYLEY'S industrial oil and fat products. $5^{\text {th }}$ ed. New York: John Wiley, 1996, v.1. Cap.10.

HWANG, S.T.; KAMMERMEYER, K. Membranes in Separations. In: CHERYAN,M. (Ed.). Ultrafiltration and microfiltration handbook. Chicago: Technomic Publ., 1998. 526p.

IWAMA, A. New process for purifying soybean oil by membrane separation and an economical evaluation of the process. Journal of American Oil Chemists' Society, Champaign, v.64, n.9, p.244-250, 1987.

KALE, V.; KATIKANENI, S.P.R.; CHERYAN, M. Deacidifying rice bran oil by solvent extraction and membrane technology. Journal of American Oil Chemists' Society, Champaign ,v.76, n.6, p.723-727, 1999.

KIM, I. et al. Phospholipids separation (degumming) from crude vegetable oil by polyimide ultrafiltration membrane. Journal of Membrane Science, Amsterdan, v.205, p.113-123, 2002. 
KOIKE, S. et al. Separation of oil constituents in organic solvents using polymeric membranes. Journal of American Oil Chemists' Society, Champaign, v. 79, n.9, p.937-942, 2002.

22 KORIS, A.; VATAI, G. Dry degumming of vegetable oils by membrane filtration. Desalination, Amsterdan, v. 148, p.149-153, 2002.

23 KOSEOGLU, S.S. Membrane technology for edible oil refining. Oils \& Fats International, Surrey, v. 5, p.16-21, 1991.

24 KOSEOGLU, S.S; ENGELGAU, D.E. Membrane applications and research in edible oil industry: an assessment. Journal of American Oil Chemists' Society, Champaign ,v.67, n.4, p.239-249, 1990.

KOSEOGLU, S.S.; RHEE,K.C.; LUSAS, E.W. Membrane processing of crude vegetables oils: laboratory scale membrane degumming, refining and bleaching. Journal of American Oil Chemists' Society, Champaign, v.27, n.6, p.182-188, 1989.

KUMAR, K. N.; BHOWMICKD, N. Separation of fatty acids/triacylglicerols by membrane. Journal of American Oil Chemists' Society, Champaign, v.73, n.3, p.399-401, 1996.

27 LIN, L.; RHEE, K.C.; KOSEOGLU, S.S. Bench-scale membrane degumming of crude vegetable oil: process optimization. Journal of Membrane Science, Amsterdan, v.134, p.101-108, 1997.

MODLER, W.H. Milk Processing. In: SHURYO, N.; MODLER, W.H. Food protein processing applications. New York: Wiley, 2000. p.1-88.

MOURA, J.M.L.N. et al. Degomagem de óleo de soja bruto por ultrafiltração. In: CONGRESSO IBERO-AMERICANO EM CIÊNCIA E TECNOLOGIA DE MEMBRANAS, 4., Florianópolis, SC, 2003. Resumos... Florianópolis: Imprensa da Universidade Federal de Santa Catarina, 2003.

30 NAGESHA, G.K.; SUBRAMANIAN, R.; SANKAR, K.U. Processing of tocopherol and FA systems using a nonporous denser polymeric membrane. Journal of American Oil Chemists' Society, Champaign, v.80, n.4, p.397-402, 2003.

$31 \quad \mathrm{OCHOA}, \mathrm{N}$. et al. Ultrafiltration of vegetable oils degumming by polymeric membranes. Separation and Purification Technology, Amsterdan, v.2223, p.417-422, 2001.

OSTERGAARD, B. Applications of membrane processing in the dairy industry. In: CONCENTRATION and drying of foods. Amsterdan: Elsevier, 1989. p.133145.

PAGLIERO, C. et al. Degumming of crude soybean oil by ultrafiltration using polymeric membranes. Journal of American Oil Chemists' Society, Champaign, v.78, n.8, p.793 -796, 2001.

PAGLIERO, C. et al. Aplicación de membranas para el desgomado de 
aceite crudo de soja y girasol. In: CONGRESSO IBERO-AMERICANO EM CIÊNCIA E TECNOLOGIA DE MEMBRANAS 4, Florianópolis, 2003. Resumos... Florianópolis: Imprensa da Universidade Federal de Santa Catarina, 2003.

PAGLIERO, C. et al. Vegetable oil degumming with polyimide and polyvinylidenefluoride ultrafiltration membranes. Journal of Chemical Technology and Biotechnology, West Sussex, v.79, p.148-152, 2004.

PARDUN, H. Gewinnung, Eigenschaften, Verarbeitung und Anwendung pflanzlicher Phosphatidpräparate. In: DIE PFLANZENLECITHINE. Augsburg: Ed. Hafen-Mühlen-Werke, 1988. 559 p.

PAULSON, D.J.; WILSON,R.L.; SPATZ,D.D. Crossflow membrane technology and its applications. Food Technology, Chicago, v.12, p.77-87, 1984.

PORTER, M.C. Ultrafiltration. In: PORTER, M.C. (Ed.) Handbook of industrial membrane technology. New Jersey: Noyes Publications, 1990.

REDDY, K. K.; NAKAJIMA, M. Membrane decolorization of crude oil. In: INTERNATIONAL CONGRESS ON MEMBRANES. Yokohama, Japan. Proceedinds... Amsterdan: Ed.Elsevier, 1996. p. 894.

REDDY, K. et al. Decolorization of vegetable oils by membrane processing. European Food Research Technology, New York, v.213, p.212-218, 2001.

RENNER, E.; SALAM, M.H.A. Basic principles. In: RENNER, E.; SALAM, M.H.A ed. Application of ultrafiltration in the industry. London: Elsevier Science, 1991. p. 7-29.

ROSENBERG, M. Current and future applications for membrane process in the dairy industry. Trends in Food Science and Technology, Cambridge, v.6, p.12-19,1995.

RYDBERG, J.; MUSIKAS,C.; CHOPPIN, G.R. Principles and practices of solvent extraction. New York: Marcel Dekker 1992. 584 p.

SNAPE, J.B.; NAKAJIMA, M. Processing of agricultural fats and oils using membrane technology. Journal of Food Engineering, Essex, v.30, p.1-41, 1996.

SOARES, M.S. et al. Deodorization of soybean oil degummed by ultrafiltration using ceramic membrane. In: WORLD SOYBEAN RESEARCH CONFERENCE, 6., Foz do Iguaçu, 2004. Abstracts... Londrina: Ed. Embrapa Soja, 2004.

STRATHMANN, H. Synthetic membranes and their preparation. In: PORTER, M.C. (Ed.) Handbook of industrial membrane technology. New Jersey: Noyes Publications, 1990.

SUBRAMANIAN, R. et al. Membrane process for premium quality expellerpressed vegetable oils. Food Research International, v.31, n.8, p.587593, 1998. 
48 SUBRAMANIAN, R. et al. Characterization of phospholipid reverse micelles in relation to membrane processing of vegetable oils. Eur. J. Lipid Sci. Technol, Weinheim, v.103, p.93-97, 2001a.

49 SUBRAMANIAN, R. et al. Rejection of carotenoids in oil systems by a nonporous polymeric composite membranes. Journal of American Oil Chemists' Society, Champaign, v.78, n.8, p.803 -807, 2001b.

50 SUBRAMANIAN, R. et al. Application of dense membrane theory for differential permeation of vegetable oil constituents. Journal of Food Engineering, Essex, v.60, p.249-256, 2003.

51 SUBRAMANIAN, R.; NAKAJIMA, M. Membrane degumming of crude soybean and rapessed oils. Journal of American Oil Chemists' Society, Champaign, v.74, n.8, p.971-975, 1997

52 SUBRAMANIAN, R.; NAKAJIMA, M.; KAWAKATSU, T. Processing of vegetable oils using polymeric composite membranes. Journal of Food Engineering, Essex, v.38, p.41-56, 1998.

53 SUTHERLAND, J.; FREE, M.J. A method for monitoring leakage through membranes during ultrafiltration of milk. Journal Dairy Technology, Austr, v.46, n.1 p.40-42, 1991.

54 USDA, 2004. Foreign Agricultural Service. Census of Agriculture: crops and vegetable oils in the world. Disponível em: <www.nass.usda.gov/ census/census02/volume1/us/index2.htm>. Acesso em 06 abril 2004.

55 ZANINI, J.M. Reaproveitamento de águas de lavagem das lavadoras de garrafas das indústrias de bebidas. Engarrafador Moderno, Santo André, v.36, p.8-13, 1994.

56 ZWIJNENBERG, H.J. et al. Acetone-stable nanofiltration membranes in deacidifying vegetable oil. Journal of American Oil Chemists' Society, Champaign, v.76, n.1, p.83 -87, 1999. 